\title{
Intestinal tuft cells: epithelial sentinels linking luminal cues to the immune system
}

\author{
F Gerbe ${ }^{1,2,3}$ and P Jay ${ }^{1,2,3}$
}

Epithelial tuft cells (also known as "brush" cells in the airway) were first identified morphologically, almost six decades ago in the trachea and gastro-intestinal tract, but their function remained mysterious until three almost simultaneous reports recently revealed their essential role in the initiation of immune type 2 responses. This is a new and exciting example of cooperation between the epithelial and haematopoietic compartments for the management of enteric parasite infections. Here we review tuft cell functions and markers, and anchors epithelial tuft cells within the current paradigm of type 2 immune responses.

\section{CELLULAR COMPOSITION OF THE INTESTINAL MUCOSA}

The intestinal mucosa has two principal components: an epithelial cell monolayer and an underlying lamina propria (Figure 1).

The lamina propria is separated from the epithelial monolayer by a basement membrane and it is constituted of a very diverse populations of cells, including among others mesenchymal cells, such as fibroblasts, myo-fibroblasts, pericytes, endothelial and smooth muscle cells, as well as hematopoietic immune cells such as B and T lymphocytes, dendritic cells, and macrophages. ${ }^{1}$ The large amount of immune cell infiltrate present in the lamina propria critically contributes to the barrier function of the adjacent monolayer of epithelial cells that separates our organism from its "environment" present in the gut lumen. ${ }^{2,3}$

\section{THE EPITHELIAL CRYPT: A PROLIFERATION COMPARTMENT}

The intestinal epithelium, the fastest renewing structure of our bodies, is organized as two spatially and functionally distinct compartments, the crypt and the villus. ${ }^{4}$ The crypt compartment is organized as a bottle-shaped invagination into the underlying lamina propria. Crypts contain the stem cells, located at their base and, above the stem cell zone, the highly proliferating transit amplifying zone, responsible for the generation of sufficient cells to completely renew the epithelium within a week. ${ }^{5}$ The emerging picture is that crypt base-located columnar stem cells expressing the leucine-rich repeat containing $\mathrm{G}$ protein-coupled receptor 5 (Lgr5) can selfrenew and generate all the differentiated cell types of the epithelium, both in vivo ${ }^{6}$ and in ex vivo organoid cultures. ${ }^{7}$ Just above the stem cell zone, the so-called +4 cell position, four cell rows above the crypt base, has long been thought to correspond to a distinct stem cell population, with slower proliferation rates, that may constitute a reserve stem cell pool capable of reconstituting the $\operatorname{Lgr} 5^{+}$crypt base-located columnar cells after injury. ${ }^{8,9}$ However, other studies based on lineage tracing from $\mathrm{Dll}^{+}$progenitor cells provided evidence that this position is occupied by early differentiating secretory cells. Dll1 ${ }^{+}$cells and the abundant absorptive enterocyte progenitors expressing the intestinal alkaline phosphatase $(A l p i)$ gene, both have the capacity to revert to the stem cell state upon injury of the crypt base-located columnar stem cell compartment. ${ }^{10,11}$ Thus, a picture is emerging in which cell plasticity could have important roles in crypt homeostasis and account for some of the heterogeneity of cells with stem cell capacity.

Crypts also contain Paneth cells, a terminally differentiated cell type intermingled with stem cells at the crypt base. Paneth cells are the only long-lived differentiated intestinal epithelial cell, ${ }^{12}$ and they function as regulators of the microbial communities present in the gut, and as a niche for the stem cells. $^{13}$ 


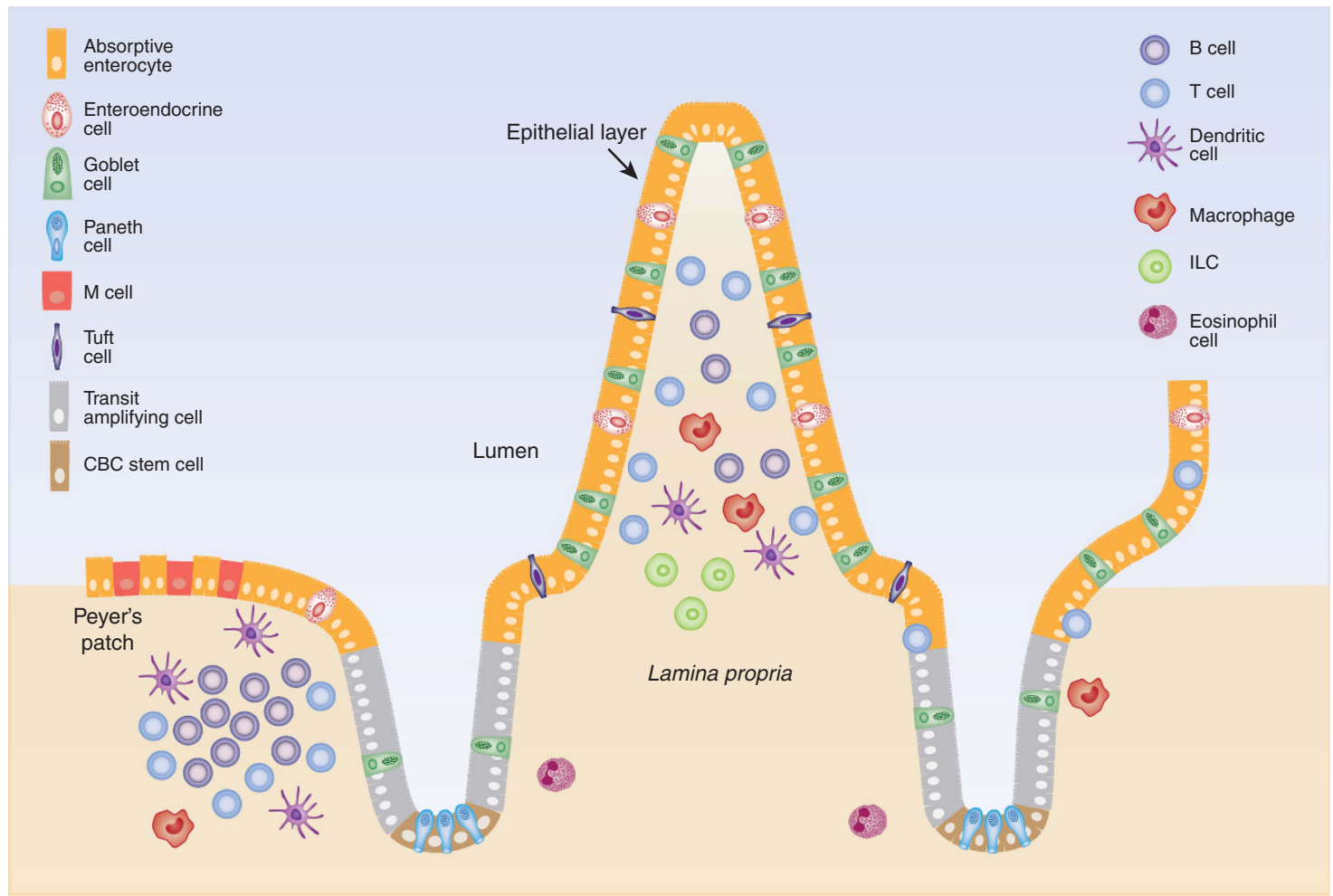

Figure 1 Schematic view of the intestinal epithelium and underlying immune cells. Epithelial crypts contain stem cells and their progeny that migrates through the transit-amplifying compartment, and differentiated Paneth cells. Epithelial villi contain only non-proliferative, differentiated, cells, including enterocytes, goblet, enteroendocrine and tuft cells. The lamina propria compartment contains different types of hematopoietic cells, depending on the immune status.

\section{THE EPITHELIAL VILLUS COMPARTMENT CONTAINS SPECIALIZED CELL TYPES}

The progeny of the stem cells migrates toward the upper crypt, where they differentiate into specialized cell types before populating the neighboring villi and eventually they undergo anoikis and desquamate in the gut lumen when they reach the villus tip. Until recently, three specialized cell types were usually mentioned in the villus compartment, including nutrientabsorbing enterocytes, mucus-secreting goblet cells and the entero-endocrine cells that produce several hormones that regulate gut physiology. ${ }^{14}$ In specific areas of the epithelium, called Peyer's patches, progenitor cells differentiate into specialized "M cells" that are capable of internalizing luminal antigens and presenting them to lymphocytes. ${ }^{15}$ In addition, it has long been known that additional cell types are present in the intestinal epithelium, including cup cells ${ }^{16}$ (although described only in guinea pigs, monkeys and rabbits) and the tuft cells, also known as brush, caveolated, multivesicular, fibrillovesicular, agranular light, or solitary chemosensory cells. ${ }^{17}$

\section{THE TUFT CELL TYPE: FROM A MORPHOLOGY-BASED IDENTIFICATION TO A SPECIFIC MARKER SIGNATURE}

Without extensively describing all the tuft cell markers that are reviewed elsewhere, ${ }^{18}$ it is interesting to recall the successive discoveries of some of these markers and their impact on the functional understanding of intestinal tuft cells. Tuft cells were initially identified in the trachea and gastro-intestinal tract by electron microscopy thanks to their unique morphology, with a unique tubulovesicular system and an apical bundle of microfilaments connected to a tuft of long and thick microvilli projecting into the lumen. ${ }^{19-21}$ It is therefore not surprising that tuft cells were first characterized by the expression of structural markers. These include proteins related to their apical brush border such as Villin, ${ }^{22}$ expression of specific cytokeratins including Krt18, ${ }^{23}$ and proteins of their microtubule network such as alpha-tubulin and its acetylated form. ${ }^{24}$ However, none of these markers has a strictly tuft cell-restricted expression and the unambiguous identification of tuft cells remained difficult by routine techniques. When it was discovered that tuft cells share morphological and likely also functional features with taste-receptor cells, ${ }^{25-27}$ the set of tuft cell markers was enriched with new proteins related to the chemosensory attributes of tuft cells. These latter proteins notably include $\alpha$-gustducin, ${ }^{25}$ as well as the cation channel Trpm5. ${ }^{26,28}$ Retrospectively, the demonstration of Trpm5 expression in tuft cells could be considered as a great breakthrough since this protein is now known to have essential roles in the function of tuft cells. ${ }^{29,30}$ Moreover, the generation of a Trpm5-eGFP reporter allele led to the discovery of most of the currently known tuft cell markers, ${ }^{27}$ including transcription factors, eicosanoids biosynthesis pathway components and type 2 immune response-related genes. Most of these markers have been validated in either the intestinal $^{31}$ or gastric mucosa ${ }^{24}$ or even in pancreatic pr,33 $^{32,3}$ tissues. The microtubule-linked protein kinase 1 (Dclk1), 

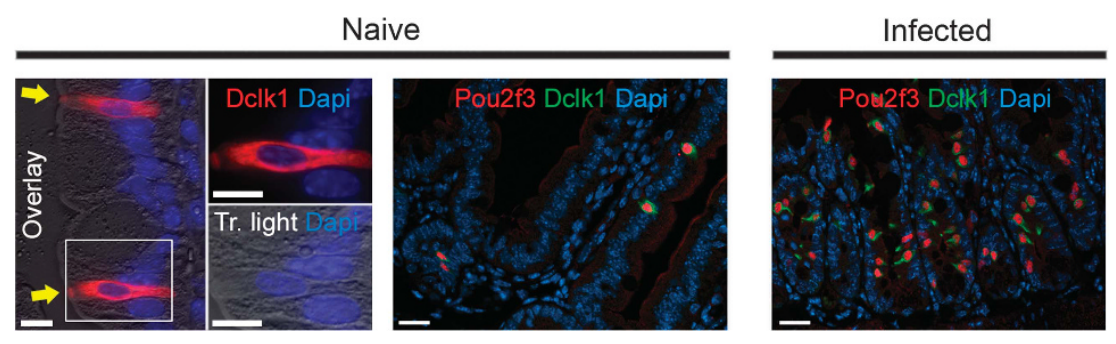

Figure 2 Tuft cell detection using two molecular markers reveals strong expansion of the tuft cell lineage during enteric parasitic infection. Pictures show tuft cells in the intestinal epithelium of naive mice, stained for detection of Dclk1 (left panel) or Dclk1 and Pou2f3 (middle panel). The latter also indicates the basal representation of the tuft cells within the intestinal epithelium. The right panel shows the expansion of Dlk1-and Pou2f3-expressing tuft cells 7 days after infection with the Nippostrongilus brasiliensis helminth.

which was initially reported as a putative marker of gastric ${ }^{34}$ and intestinal ${ }^{35}$ epithelium stem cells, is actually predominantly expressed in tuft cells ${ }^{36}$ and in a rare sub-population of enteroendocrine cells expressing the insulinoma-associated 1 (Insm1) protein. ${ }^{37}$ Dclk1 is thus widely used as a tuft cell marker ${ }^{24,30,31,37-39}$ (Figure 2). Of note, the expression of the growth factor independent $1 \mathrm{~b}$ (Gfilb) transcription factor was described as specific to the tuft cell lineage in the context of the intestinal epithelium and is therefore also a useful marker. ${ }^{37}$

\section{POU2F3: A MASTER GENE FOR INTESTINAL TUFT CELL DIFFERENTIATION}

Several of the key transcription factors controlling the specification or maintenance of the different intestinal epithelial cell types have been identified. ${ }^{4}$ The Spi-1/PU.1 related (Spib) transcription factor is required for differentiation of M cells. ${ }^{40,41}$ The Atonal bHLH factor 1 (Atoh1), in turn, is essential for the specification of goblet, Paneth, and enteroendocrine cells, which are collectively known as the secretory or granulocytic cell types of the intestinal epithelium. ${ }^{42}$ The Srybox 9 (Sox9) factor, expressed in both the crypt stem and Paneth cells, ${ }^{43}$ and the Neurogenin 3 (Ngn3), expressed in crypt progenitor cells, ${ }^{44}$ are required for Paneth ${ }^{45,46}$ and enteroendocrine ${ }^{47}$ cell specification or/an differentiation, respectively. The Kruppel-like factor 4 (Klf4) is necessary for goblet cells differentiation. ${ }^{48}$ Other important regulators include the Etsdomain transcription factor Spdef for Paneth and goblet cells ${ }^{49,50}$ differentiation and the Growth factor independent-1 (Gfil) transcription factor that controls the relative representation of enteroendocrine cells compared with that of goblet and Paneth cells. ${ }^{51,52}$ Except for Atoh1, for which two separate studies came to divergent conclusions with one reporting absence of tuft cells in Atoh1-deficient mice, like goblet, Paneth, and enteroendocrine cells, ${ }^{31}$ and the other finding expansion of the tuft cell lineage in Atoh1-deficient mice, ${ }^{37}$ all the abovementioned transcription factors are dispensable for tuft cell specification. ${ }^{31}$ The role of Spib in tuft cell differentiation was not investigated.

More recently, the role of the Pou domain, class 2, transcription factor 3 (Pou2f3) transcription factor was also investigated. Pou $2 \mathrm{f3}$ expression is essentially restricted to Dclk1- and Gfilb-expressing tuft cells within the intestinal epithelium (Figure 2). ${ }^{38}$ Pou2f3-deficient mice lack all Pou2f3- expressing taste receptor cells including sweet, umami, and bitter taste cells. ${ }^{53}$ Pou2f3-deficient mice also lack Trpm5expressing chemosensory cells in the nasal cavity ${ }^{54}$ and olfactory epithelium. ${ }^{55}$ The analysis of the intestinal epithelium of Pou2f3-deficient mice revealed a complete absence of tuft cells, whereas other cell types in the intestinal epithelium were present in normal numbers. ${ }^{38}$ This study thus identified Pou $2 \mathrm{f3}$ as the first transcription factor specifically required for tuft cell presence in the intestinal epithelium (Figure 3). Pou2f3deficient mice are thus a useful model to investigate the function of the tuft cell lineage. ${ }^{38}$

\section{TUFT CELLS INITIATE TYPE 2 RESPONSES TO HELMINTH INFECTIONS}

Three independent and complementary studies ${ }^{30,38,39}$ have recently revealed another critical function of tuft cells in the initiation of type 2 immune responses, which are typically involved during intestinal protozoa or helminth parasite infections, and which are deleteriously activated in allergies. ${ }^{56}$ Type 2 responses require activation and recruitment of type 2 helper T cells (Th2 cells) and group 2 innate lymphoid cells (ILC2s) by epithelial cell-derived cytokines, including IL25, IL33 and thymic stromal lymphopoietin. ${ }^{57-59}$ Production of IL13 by Th2 cells and ILC2s causes the remodeling of the intestinal epithelium, including goblet cell hyperplasia ${ }^{60}$ and hypercontractibility of smooth muscle cells ${ }^{61}$ that peak at the time of worm expulsion.

In the Jay group study, it was found that the tuft cell population dramatically expands during infections with helminths such as Nippostrongylus brasiliensis or Heligmosomoides polygyrus, in an IL4/IL13 signaling-dependent way. Furthermore, in Pou2f3-deficient mice that lack tuft cells, the immune response was greatly altered; the mice had impaired recruitment of Th2 and ILC2 cells, reduced levels of IL13 production, nearly abolished goblet cell hyperplasia and greatly delayed worm expulsion. When investigating the link between the presence of tuft cells and efficient type 2 responses during infection, tuft cells were found to be the epithelial source of the IL25 alarmin expression. This identified tuft cells as the trigger to the induction of the type 2 response following parasite infections. ${ }^{38}$

The Locksley group reached identical conclusions using an elegant mouse model that combines an IL25 expression 


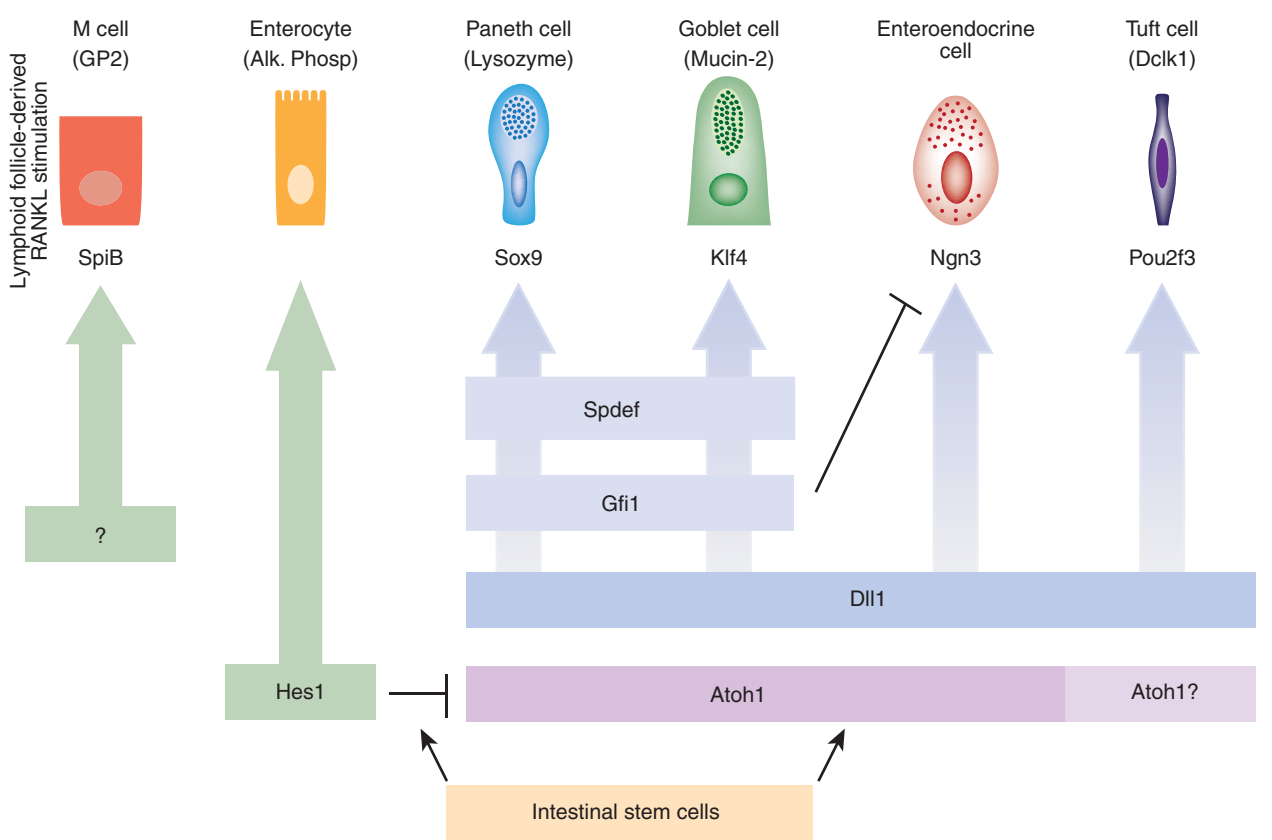

Figure 3 Genetic hierarchy controlling specification and differentiation of the different cell types constituting the intestinal epithelium. The progeny of intestinal stem cells can engage either toward an absorptive (columnar-left) or a secretory (granulocytic-right) cell fate, depending on the expression of Hairy/enhancer of split 1 (Hes1) or Atonal homologue 1 (Atoh1), respectively. The Delta-like ligand 1 (DIl-1) gene is expressed in one or several type(s) of crypt-located secretory progenitor cells from which Paneth, goblet, enteroendocrine and tuft cells derive. Among the cells of the secretory lineages, Neurogenin3 (Ngn3) is required for presence of all enteroendocrine cells. Growth factor independent 1 (Gf1) is necessary to prevent Ngn3 expression within Paneth and goblet cells, while SAM pointed domain containing ETS transcription factor (Spdef) is required for their terminal differentiation. SRY (sex determining region Y)-box 9 (Sox9) and Kruppel-like factor 4 (Klf4) are necessary for Paneth cells and goblet cells differentiation, respectively. The POU domain, class 2, transcription factor 3 gene (Pou2f3) is required for specification/differentiation of tuft cells. M-cells derive from intestinal stem cells and differentiate from Spi-B transcription factor (Spi-1/PU.1 related) (SpiB)-expressing cells, upon induction by the receptor activator of nuclear factor kappa-B ligand (RANKL) molecules produced by neighboring hematopoietic cells.

reporter and inducible deletion of the Il25 gene. The deletion of the Il25 gene led to essentially identical consequences as the absence of the tuft cell lineage, ${ }^{39}$ suggesting that IL25 production is the essential function of tuft cells, at least in the context of parasite infections.

The Garrett group study addressed the chemosensory function of tuft cells in the context of protozoa or helminth infections. Deletion of the TRPM5 cation channel, which is involved in the transduction of signals from many tastechemosensory G protein-coupled receptors, ${ }^{62}$ greatly impaired the type 2 response following infection with the Tritrichomonas muris protozoa. Trpm5-deficient mice failed to mount an efficient epithelial response and had impaired tuft cell expansion and a reduced IL25 expression and a lowered frequency of lamina propria IL17RB ${ }^{+}$ILC2s. ${ }^{30}$ This study thus identified TRPM5 as a critical player in the induction of type 2 responses, which likely act upstream of IL25 production.

Together, these three studies revealed an unexpected level of functional integration and cooperation between the epithelial and hematopoietic compartments in mounting an efficient response against parasite infections.

\section{TRPM5 AND TASTE RECEPTOR SIGNALING: TOWARD A NEW PARADIGM OF PARASITE RECOGNITION?}

The finding that cells with chemosensory pathways are critical for the initiation of type 2 immune responses to parasite infections $s^{30,38,39}$ raised the question of whether tuft cells are the direct sensors of the presence of parasites in the gut lumen. Since Trpm5 is involved in the transduction of signals from taste-chemosensory $\mathrm{G}$ protein-coupled receptors, ${ }^{62}$ this finding opens avenues toward the identification of the parasite compounds that epithelial cells detect, as well as the receptor involved in parasite recognition upstream of Trpm5. Notably, Trpm 5 transduces signals from taste receptors that respond to bitter-, sweet-, and umami-tasting substances. ${ }^{63,64} \mathrm{Trpm} 5$ also transduces signals when tuft cells react after stimulation with glucose or high salt solution, ${ }^{29}$ or with bitter compounds such as cycloheximide or denatonium. ${ }^{65,66}$ Multiple members of the T2R family of bitter taste receptors are expressed in the GI tract $^{67}$ and these proteins can respond to a wide range of chemicals with diverse specificities. ${ }^{68}$ It is therefore tempting to envision a parallelism between the sensing of microbes by the TLR- and NOD-dependent pathways, and that of multicellular parasites by Trpm5-signaling receptors such as the taste receptors (Figure 4).

\section{PERSPECTIVES ON TUFT CELL FUNCTION}

The three recent simultaneous studies of tuft cell function during parasite infections mentioned above ${ }^{30,38,39}$ bring us to a new paradigm of type 2 immune responses. Not only has the precise epithelial source of IL25 been identified, but IL25 production relies on a cell type, namely the tuft cell, that lacked 


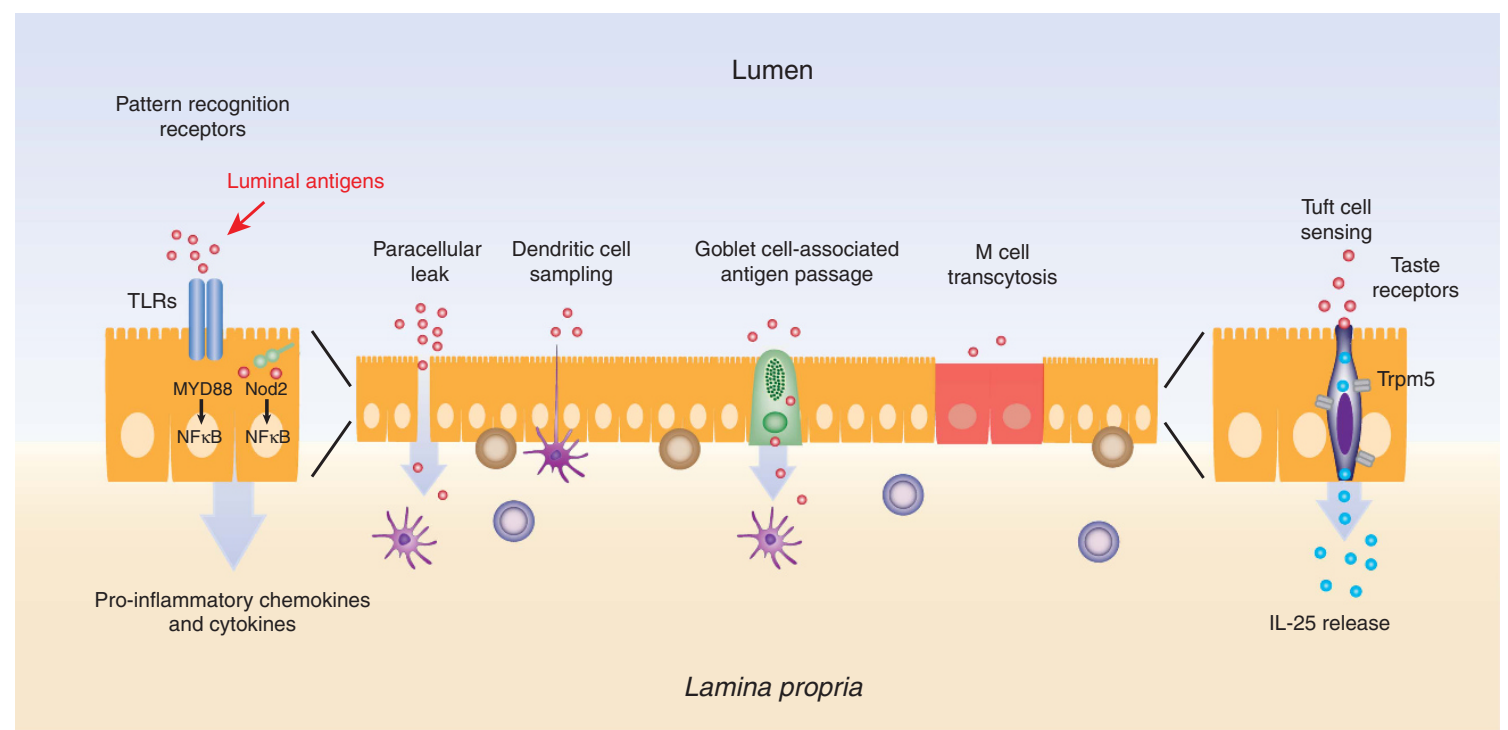

Figure 4 Multiple complementary pathways underlie the trans-epithelial communication of luminal clues to the lamina propria. Epithelial cells are known to express either membrane-bound or intra-cellular pattern recognition receptors (PRRs), including members of the Toll-like receptor (TLR) family or NOD-like receptors. TLRs are activated by a wide spectrum of ligands, including bacterial and viral nucleic acids or components, such as lipopolysaccharides, flagellin or peptidoglycans. TLR signaling involves several adapter proteins, including MyD88, which lead to the production of inflammatory cytokines. NOD-like receptors are intracellular proteins that are activated by invasive bacteria peptidoglycan and which are involved in NFkB activation. Among the processes involved in luminal sampling by lamina propria-residing cells, paracellular leakage allows passage of small molecules across an intact epithelial layer. Dendritic cells can also directly sample the luminal content through the formation of trans-epithelial dendrites. Lymphoid follicle-associated epithelium M-cells are known to act as transporter of luminal molecules via the transcytosis process, thus enabling antigenic presentation to underlying immune cells. Mucus-secreting cells, known as goblet cells, can allow passage of antigens for preferential delivery to $\mathrm{CD}_{103^{+}}$dendritic cells. ${ }^{70}$ Finally, chemosensory tuft cells are involved in type-2 immune response initiation through secretion of the IL25 alarmin by a Trmp5-dependent mechanism, which involves as yet unknown taste receptors.

a clear function thus far and that had been strikingly overlooked since its discovery almost 60 years ago. Other questions remain of course. For example, cells with a tuft cell morphology have also been identified in the airway, where they are often called brush cells. ${ }^{19,69}$ Recent studies revealed the cholinergic nature of the airway tuft/brush cells i.e., their capacity to express acetylcholine and the vesicular acetylcholine transporter VAChT. These cells also express a bitter taste transduction system, including T2R taste receptors, the G-protein alphagustducin, PLC- $\beta 2$ enzyme and the transient receptor potential cation-channel Trpm5 ${ }^{28,65}$ Cholinergic tuft/brush cells establish contacts with peptidergic cholinoceptive vagal sensory neurons and application of a bitter substance to tracheal mucosa resulted in cholinergically driven aversive respiratory reflexes, i.e., a reduction of respiratory rates. ${ }^{49,50}$ Airway tuft/ brush cells thus link the luminal chemical composition of the lower airway to the control of breathing. However, are the tuft/ brush cells from the airway the same cell type as the intestinal tuft cell? Is their similarity limited to a related morphology and the expression of a couple of markers, or do they share the same Pou2f3-dependent differentiation pathway and identical functions? Since different roles have been found so far in airway tuft/ brush vs. intestinal tuft cells, answers to these questions may reveal additional functions for these cells. Even in a single organ such as the intestine, whether the function of proximally located tuft cells is identical to that of more distally-, or colon-located, tuft cells is still not certain. Finally, the discovery of the role of intestinal tuft cells in regulating type 2 immune responses during parasite infections means it will be important to investigate their role in other type 2-dependent processes (such as allergy) in both the airway and in the gastro-intestinal epithelia. From the initial discovery of tuft cells, it took almost 60 years to identify their first functions. We anticipate rapid development in the understanding of tuft cell functions with the reliable molecular markers and dedicated mouse models now available.

\section{ACKNOWLEDGMENTS}

We thank the Institut National de la Santé et de la Recherche Médicale, the Centre National de la Recherche Scientifique, the Association pour la Recherche contre le Cancer (SL220110603456 to P.J.), Agence Nationale de la Recherche (ANR-09-BLAN-0368-01 and ANR-14-CE14-0025-01) and Institut National du Cancer (INCa 2014-174) for support.

\section{AUTHOR CONTRIBUTIONS}

F.G. and P.J. both contributed to the writing of the text and the preparation of the figures.

\section{DISCLOSURE}

The authors declared no conflict of interest.

c) 2016 Society for Mucosal Immunology

\section{REFERENCES}

1. Hunyady, B., Mezey, E. \& Palkovits, M. Gastrointestinal immunology: cell types in the lamina propria-a morphological review. Acta Physiol. Hung. 87, 305-328 (2000). 
2. Peterson, L.W. \& Artis, D. Intestinal epithelial cells: regulators of barrier function and immune homeostasis. Nat. Rev. Immunol. 14, 141-153 (2014).

3. Mowat, A.M. \& Agace, W.W. Regional specialization within the intestinal immune system. Nat. Rev. Immunol. 14, 667-685 (2014).

4. van der Flier, L.G. \& Clevers, H. Stem cells, self-renewal, and differentiation in the intestinal epithelium. Annu. Rev. Physiol. 71, 241-260 (2009).

5. Clevers, $H$. The intestinal crypt, a prototype stem cell compartment. Cell 154, 274-284 (2013).

6. Barker, N. et al. Identification of stem cells in small intestine and colon by marker gene Lgr5. Nature 449, 1003-1007 (2007).

7. Sato, T. et al. Single Lgr5 stem cells build crypt-villus structures in vitro without a mesenchymal niche. Nature 459, 262-265 (2009).

8. Tian, $\mathrm{H}$. et al. A reserve stem cell population in small intestine renders Lgr5-positive cells dispensable. Nature 478, 255-259 (2011).

9. Sangiorgi, E. \& Capecchi, M.R. Bmi1 is expressed in vivo in intestinal stem cells. Nat. Genet. 40, 915-920 (2008).

10. Es, J. H. van et al. Dll1 (+) secretory progenitor cells revert to stem cells upon crypt damage. Nat. Cell Biol. 14, 1099-1104 (2012).

11. Tetteh, P.W. et al. Replacement of lost Lgr5-positive stem cells through plasticity of their enterocyte-lineage daughters. Cell Stem Cell 18, 203-213 (2016).

12. Ireland, H., Houghton, C., Howard, L. \& Winton, D.J. Cellular inheritance of a Cre-activated reporter gene to determine Paneth cell longevity in the murine small intestine. Dev. Dyn. 233, 1332-1336 (2005).

13. Sato, T. et al. Paneth cells constitute the niche for Lgr5 stem cells in intestinal crypts. Nature 469, 415-418 (2011).

14. Parker, H.E., Gribble, F.M. \& Reimann, F. The role of gut endocrine cells in control of metabolism and appetite. Exp. Physiol. 99, 1116-1120 (2014).

15. Neutra, M.R., Frey, A. \& Kraehenbuhl, J.P. Epithelial M cells: gateways for mucosal infection and immunization. Cell 86, 345-348 (1996).

16. Madara, J.L. Cup cells: structure and distribution of a unique class of epithelial cells in guinea pig, rabbit, and monkey small intestine. Gastroenterology 83, 981-994 (1982).

17. Luciano, L., Reale, E. \& Ruska, H. [On a glycogen containing brusc cell in the rectum of the rat]. Z Zellforsch Mikrosk Anat 91, 153-158 (1968).

18. Gerbe, F., Legraverend, C. \& Jay, P. The intestinal epithelium tuft cells: specification and function. Cell. Mol. Life Sci. 69, 2907-2917 (2012).

19. Rhodin, J. \& Dalhamn, T. Electron microscopy of the tracheal ciliated mucosa in rat. Z. Zellforsch. Mikrosk. Anat. 44, 345-412 (1956).

20. Jarvi, O. \& Keyrilainen, O. On the cellular structures of the epithelial invasions in the glandular stomach of mice caused by intramural application of 20-methylcholantren. Acta Pathol. Microbiol. Scand. Suppl. 39, 72-73 (1956).

21. Sato, A. Tuft cells. Anat. Sci. Int. 82, 187-199 (2007).

22. Hofer, D. \& Drenckhahn, D. Identification of brush cells in the alimentary and respiratory system by antibodies to villin and fimbrin. Histochemistry 98 , 237-242 (1992).

23. Hofer, D. \& Drenckhahn, D. Cytoskeletal markers allowing discrimination between brush cells and other epithelial cells of the gut including enteroendocrine cells. Histochem. Cell Biol. 105, 405-412 (1996).

24. Saqui-Salces, M. et al. Gastric tuft cells express DCLK1 and are expanded in hyperplasia. Histochem. Cell Biol. 136, 191-204 (2011).

25. Hofer, D., Puschel, B. \& Drenckhahn, D. Taste receptor-like cells in the rat gut identified by expression of alpha-gustducin. Proc. Natl Acad. Sci. USA 93, 6631-6634 (1996).

26. Bezencon, C., le Coutre, J. \& Damak, S. Taste-signaling proteins are coexpressed in solitary intestinal epithelial cells. Chem. Senses 32, 41-49 (2007).

27. Bezencon, C. et al. Murine intestinal cells expressing Trpm5 are mostly brush cells and express markers of neuronal and inflammatory cells. J. Comp. Neurol. 509, 514-525 (2008).

28. Kaske, S. et al. TRPM5, a taste-signaling transient receptor potential ion-channel, is a ubiquitous signaling component in chemosensory cells. BMC Neurosci. 8, 49 (2007).

29. Kokrashvili, Z. et al. Release of endogenous opioids from duodenal enteroendocrine cells requires Trpm5. Gastroenterology 137, 598-606 (2009).

30. Howitt, M.R. et al. Tuft cells, taste-chemosensory cells, orchestrate parasite type 2 immunity in the gut. Science 351, 1329-1333 (2016).
31. Gerbe, F. et al. Distinct ATOH1 and Neurog3 requirements define tuft cells as a new secretory cell type in the intestial epithelium. J. Cell Biol. 192, 767-780 (2011).

32. Bailey, J.M. et al. DCLK1 marks a morphologically distinct subpopulation of cells with stem cell properties in preinvasive pancreatic cancer. Gastroenterology 146, 245-256 (2013).

33. Delgiorno, K.E. et al. Identification and manipulation of biliary metaplasia in pancreatic tumors. Gastroenterology 146, 233-44.e5 (2013).

34. Giannakis, M. et al. Molecular properties of adult mouse gastric and intestinal epithelial progenitors in their niches. J. Biol. Chem. 281, 11292-11300 (2006).

35. May, R. et al. Identification of a novel putative gastrointestinal stem cell and adenoma stem cell marker, doublecortin and CaM kinase-like-1, following radiation injury and in adenomatous polyposis coli/multiple intestinal neoplasia mice. Stem Cells 26, 630-637 (2008).

36. Gerbe, F., Brulin, B., Makrini, L., Legraverend, C. \& Jay, P. DCAMKL-1 expression identifies tuft cells rather than stem cells in the adult mouse intestinal epithelium. Gastroenterology 137, 2179-2180 (2009).

37. Bjerknes, M. et al. Origin of the brush cell lineage in the mouse intestinal epithelium. Dev. Biol. 362, 194-218 (2012).

38. Gerbe, F. et al. Intestinal epithelial tuft cells initiate type 2 mucosal immunity to helminth parasites. Nature 529, 226-230 (2016).

39. von Moltke, J., Ji, M., Liang, H.-E. \& Locksley, R.M. Tuft-cell-derived IL-25 regulates an intestinal ILC2-epithelial response circuit. Nature 529, 221-225 (2015).

40. de Lau, W. et al. Peyer's patch M cells derived from Lgr5(+) stem cells require SpiB and are induced by RankL in cultured 'miniguts'. Mol. Cell Biol. 32, 3639-3647 (2012).

41. Kanaya, T. et al. The Ets transcription factor Spi-B is essential for the differentiation of intestinal microfold cells. Nat. Immunol. 13, 729-736 (2012).

42. Shroyer, N.F. et al. Intestine-specific ablation of mouse atonal homolog 1 (Math1) reveals a role in cellular homeostasis. Gastroenterology 132, 2478-2488 (2007).

43. Blache, P. et al. SOX9 is an intestine crypt transcription factor, is regulated by the Wnt pathway, and represses the CDX2 and MUC2 genes. J. Cell Biol. 166, 37-47 (2004).

44. Bjerknes, M. \& Cheng, H. Neurogenin 3 and the enteroendocrine cell lineage in the adult mouse small intestinal epithelium. Dev. Biol. 300, 722-735 (2006).

45. Bastide, P. et al. Sox9 regulates cell proliferation and is required for Paneth cell differentiation in the intestinal epithelium. J. Cell Biol. 178, 635-648 (2007).

46. Mori-Akiyama, Y. et al. SOX9 is required for the differentiation of paneth cells in the intestinal epithelium. Gastroenterology 133, 539-546 (2007).

47. Jenny, M. et al. Neurogenin3 is differentially required for endocrine cell fate specification in the intestinal and gastric epithelium. EMBO J. 21, 6338-6347 (2002).

48. Katz, J.P. et al. The zinc-finger transcription factor Klf4 is required for terminal differentiation of goblet cells in the colon. Development 129, 2619-2628 (2002).

49. Gregorieff, A. et al. The ets-domain transcription factor Spdef promotes maturation of goblet and paneth cells in the intestinal epithelium. Gastroenterology 137, 1333-45 e1-3 (2009).

50. Noah, T.K., Kazanjian, A., Whitsett, J. \& Shroyer, N.F. SAM pointed domain ETS factor (SPDEF) regulates terminal differentiation and maturation of intestinal goblet cells. Exp. Cell Res. 316, 452-465 (2009).

51. Shroyer, N.F., Wallis, D., Venken, K.J., Bellen, H.J. \& Zoghbi, H.Y. Gfi1 functions downstream of Math1 to control intestinal secretory cell subtype allocation and differentiation. Genes Dev. 19, 2412-2417 (2005).

52. Bjerknes, M. \& Cheng, H. Cell lineage metastability in Gfi1-deficient mouse intestinal epithelium. Dev. Biol. 345, 49-63 (2010).

53. Matsumoto, I., Ohmoto, M., Narukawa, M., Yoshihara, Y. \& Abe, K. Skn-1a (Pou2f3) specifies taste receptor cell lineage. Nat. Neurosci. 14, 685-687 (2011).

54. Ohmoto, M. et al. Pou2f3/Skn-1a is necessary for the generation or differentiation of solitary chemosensory cells in the anterior nasal cavity. Biosci. Biotechnol. Biochem. 77, 2154-2156 (2013).

55. Yamaguchi, T. et al. Skn-1a/Pou2f3 is required for the generation of Trpm5-expressing microvillous cells in the mouse main olfactory epithelium. BMC Neurosci. 15, 13 (2014). 
56. Allen, J.E. \& Maizels, R.M. Diversity and dialogue in immunity to helminths. Nat. Rev. Immunol. 11, 375-388 (2011).

57. Moro, K. et al. Innate production of $\mathrm{T}(\mathrm{H}) 2$ cytokines by adipose tissue-associated c-Kit(+)Sca-1(+) lymphoid cells. Nature 463, 540-544 (2010).

58. Neill, D.R. et al. Nuocytes represent a new innate effector leukocyte that mediates type-2 immunity. Nature 464, 1367-1370 (2010).

59. Fallon, P.G. et al. Identification of an interleukin (IL)-25-dependent cell population that provides IL-4, IL-5, and IL-13 at the onset of helminth expulsion. J. Exp. Med. 203, 1105-1116 (2006).

60. Herbert, D.R. et al. Intestinal epithelial cell secretion of RELM-beta protects against gastrointestinal worm infection. J. Exp. Med. 206, 2947-2957 (2009).

61. Castro, G.A., Badial-Aceves, F., Smith, J.W., Dudrick, S.J. \& Weisbrodt, N.W. Altered small bowel propulsion associated with parasitism. Gastroenterology 71, 620-625 (1976).

62. Kinnamon, S.C. Taste receptor signalling-from tongues to lungs. Acta Physiol. Oxf. Engl 204, 158-168 (2012).
63. Zhang, Y. et al. Coding of sweet, bitter, and umami tastes: different receptor cells sharing similar signaling pathways. Cell 112, 293-301 (2003).

64. Damak, S. et al. Trpm5 null mice respond to bitter, sweet, and umami compounds. Chem. Senses 31, 253-264 (2006).

65. Krasteva, G. et al. Cholinergic chemosensory cells in the trachea regulate breathing. Proc. Natl Acad. Sci. USA 108, 9478-9483 (2011).

66. Schütz, B. et al. Chemical coding and chemosensory properties of cholinergic brush cells in the mouse gastrointestinal and biliary tract. Front. Physiol. 6, 87 (2015).

67. Wu, S.V. et al. Expression of bitter taste receptors of the T2R family in the gastrointestinal tract and enteroendocrine STC-1 cells. Proc. Natl Acad. Sci. USA 99, 2392-2397 (2002).

68. Meyerhof, W. et al. The molecular receptive ranges of human TAS2R bitter taste receptors. Chem. Senses 35, 157-170 (2010).

69. Reid, L. et al. The mysterious pulmonary brush cell: a cell in search of a function. Am. J. Respir. Crit. Care Med. 172, 136-139 (2005).

70. McDole, J.R. et al. Goblet cells deliver luminal antigen to CD103+ dendritic cells in the small intestine. Nature 483, 345-349 (2012). 